\title{
BER PERFORMANCE DEGRADATION OF MC-DS-CDMA SYSTEMS JOINTLY AFFECTED BY TRANSMITTER HPA AND RECEIVER CFO IN FREQUENCY SELECTIVE FADING CHANNELS
}

\author{
Paolo Banelli, Luca Rugini \\ DIEI - University of Perugia - Via G. Duranti 93 - 06125 Perugia - Italy
}

\begin{abstract}
Multicarrier systems are highly sensitive to the intermodulation distortion (IMD) introduced by high-power amplifiers (HPA) at the transmitter and to the intercarrier interference (ICI) due to a carrier frequency offset (CFO) at the receiver. We analyze the bit-error rate (BER) degradation induced by these impairments in the downlink of a multicarrier direct-sequence code-division multiple-access (MC-DS-CDMA) systems in frequency-selective Rayleigh fading channels.
\end{abstract}

\section{INTRODUCTION}

Multicarrier CDMA techniques are attractive for future mobile broadband communications [1] because they can combine the low complexity equalization of cyclic prefixed OFDM systems, and the multiple-access interference (MAI) mitigation capabilities offered by CDMA systems.

The high sensitivity to frequency synchronization errors is one of the main problems of multicarrier schemes [2]. Indeed, the CFO, which models the frequency mismatch between the transmitter and receiver oscillators, originates ICI, thereby destroying the frequency-domain orthogonality of the transmitted data. Another serious impairment is the presence of a non linear HPA at the transmitter, which introduces IMD. Indeed, multicarrier signals are characterized by a high variability of their envelope, and consequently they are significantly distorted when the HPA works close to the saturation in order to optimize the power efficiency.

Herein we focus on a system that is commonly identified as MC-DS-CDMA [1], where multiple users are discriminated by spreading in the time domain., and we want to analyze the BER degradation introduced by the mentioned impairments. Specifically, we consider the BER performance in the downlink when the mobile receiver employs a low-complexity matched filter (MF) detector. Previous papers on this subjects considered either the effect of CFO [3] or the effect of the HPA [4]. The purpose of this paper is to develop a BER analysis that jointly takes into account $\mathrm{CFO}$ and nonlinear distortions in multipath fading channels.

\section{SYSTEM MODEL}

We consider the downlink of an MC-DS-CDMA system with
$N$ subcarriers, spaced by $\Delta_{f}=1 / T$, and $K$ users. The base station multiplexes $N$ symbols of each user over the $N$ subcarriers, by spreading each symbol in the time domain employing a user-dependent spreading code, which is denoted with $\mathbf{c}_{k}=\left[\begin{array}{lll}c_{k, 0} & \cdots & c_{k, G-1}\end{array}\right]^{T}$, where $G$ is the processing gain, and $\left|c_{k, g}\right|=G^{-1 / 2}$. The transmitted block, at the input of the HPA, can be expressed by

$$
\mathbf{u}_{\mathrm{IN}}[l G+g]=\mathbf{T}_{\mathrm{CP}} \mathbf{F}^{H} \mathbf{S}[l] \mathbf{c}[g],
$$

where $\mathbf{u}_{\mathrm{IN}}[l G+g]$ is a vector of dimension $P=N+L, L$ is the cyclic prefix length, $\mathbf{T}_{\mathrm{CP}}$ is the $P \times N$ cyclic prefix insertion matrix [5], $\mathbf{F}$ is the $N \times N$ unitary FFT matrix, $\mathbf{S}[l]$ is the $N \times K$ matrix containing the data symbols, and $\mathbf{c}[g]=\left[\begin{array}{lll}c_{1, g} & \cdots & c_{K, g}\end{array}\right]^{T}$ is the vector that contains the gth chip of the codes of all the $K$ users. The $l$ th symbol $s_{n, k}[l]=[\mathbf{S}[l]]_{n, k}$ of the $k$ th user on the $n$th subcarrier, is drawn from the 4-QAM constellation, and all the symbols are assumed to be i.i.d. with power $\sigma_{\mathrm{S}}^{2}$.

The HPA is modeled as a memoryless device by means of its $\mathrm{AM} / \mathrm{AM}$ and $\mathrm{AM} / \mathrm{PM}$ curves. By using the Bussgang theorem and the index $i=l G+g$, the transmitted block, at the output of the HPA, can be expressed as [6]

$$
\mathbf{u}_{\mathrm{OUT}}[i]=\alpha \mathbf{u}_{\mathrm{IN}}[i]+\overline{\mathbf{v}}_{\mathrm{IMD}}[i] \text {, }
$$

where $\alpha$ and the autocorrelation function $\mathbf{R}_{\overline{\mathbf{v}}}\left(g, g^{\prime}\right)$ of $\overline{\mathbf{v}}_{\text {IMD }}[i]$ depend on the AM/AM and AM/PM curves of the HPA and on the output back-off (OBO) to the HPA [6]. The validity of (2), based on a Gaussian distribution of $\mathbf{u}_{\mathrm{IN}}[i]$ in (1), is justified by the high number of subcarriers usually employed in multicarrier systems (e.g., $N \geq 32$ ).

After the parallel-to-serial conversion, the signal stream $u_{\mathrm{OUT}}[i P+p]=\left[\mathbf{u}_{\mathrm{OUT}}[i]\right]_{p+1}, \quad p=0, \ldots, P-1$, is transmitted through a multipath FIR channel $h[b]$, characterized by paths with Rayleigh statistics and maximum delay spread smaller than the cyclic prefix duration. We assume that timing information is available at the receiver. In the presence of CFO, the received stream can be expressed as [7]

$$
x[a]=e^{j 2 \pi f_{0} a T / N} \sum_{b=0}^{L} h[b] u_{\mathrm{OUT}}[a-b]+x_{\mathrm{WG}}[a],
$$

where $f_{0}$ is the CFO, and $x_{\mathrm{WG}}[a]$ represents the AWGN, with $a=i P+p$. The $P$ received samples relative to the $g$ th chip of the $l$ th symbol are grouped to form the vector $\mathbf{x}[i]=\mathbf{x}[l G+g]$, thus obtaining [7]

$$
\mathbf{x}[i]=e^{j 2 \pi \varepsilon i P / N} \tilde{\mathbf{D}}\left(\mathbf{H}_{0} \mathbf{u}_{\mathrm{OUT}}[i]+\mathbf{H}_{1} \mathbf{u}_{\mathrm{OUT}}[i-1]\right)+\mathbf{x}_{\mathrm{WG}}[i],
$$

where $[\mathbf{x}[i]]_{p+1}=x[i P+p], \varepsilon=f_{0} T$ is the normalized CFO, 
$\tilde{\mathbf{D}}$ is a $P \times P$ diagonal matrix, defined by $[\tilde{\mathbf{D}}]_{p, p}=\exp (j 2 \pi \varepsilon(p-1) / N)$, and $\mathbf{H}_{0}$ and $\mathbf{H}_{1}$ are $P \times P$ Toeplitz matrices defined by $\left[\mathbf{H}_{0}\right]_{m, n}=h[m-n]$ and $\left[\mathbf{H}_{1}\right]_{m, n}=h[m-n+P]$, respectively [5].

If $\mathbf{R}_{\mathrm{CP}}$ denotes the cyclic prefix elimination matrix, by using (1) and (2), the vector $\mathbf{y}[l G+g]=\mathbf{y}[i]=\mathbf{R}_{\mathrm{CP}} \mathbf{x}[i]$ can be expressed as [7]

$$
\begin{array}{r}
\mathbf{y}[l G+g]=e^{j 2 \pi \varepsilon((l G+g) P+L) / N} \mathbf{D H}\left(\alpha \mathbf{F}^{H} \mathbf{S}[l] \mathbf{c}[g]\right. \\
\left.+\tilde{\mathbf{v}}_{\mathrm{IMD}}[l G+g]\right)+\mathbf{y}_{\mathrm{WG}}[l G+g],
\end{array}
$$

where $\mathbf{D}$ is the $N \times N$ diagonal matrix defined by $[\mathbf{D}]_{n, n}=\exp (j 2 \pi \varepsilon(n-1) / N), \mathbf{H}=\mathbf{R}_{\mathrm{CP}} \mathbf{H}_{0} \mathbf{T}_{\mathrm{CP}}$ is the circulant channel matrix, $\tilde{\mathbf{v}}_{\mathrm{IMD}}[l G+g]=\mathbf{R}_{\mathrm{CP}} \overline{\mathbf{v}}_{\mathrm{IMD}}[l G+g]$, and $\mathbf{y}_{\mathrm{WG}}[l G+g]$ stands for the AWGN. Since $\mathbf{H}$ is circulant, it can be expressed as $\mathbf{H}=\mathbf{F}^{H} \boldsymbol{\Lambda} \mathbf{F}$, where $\boldsymbol{\Lambda}=\operatorname{diag}(\boldsymbol{\lambda})$ is a diagonal matrix representing the frequency-domain channel $\lambda=N^{1 / 2} \mathbf{F}[h[0] \cdots h[N-1]]^{T}$.

The recovery of the transmitted data is accomplished by applying the FFT at the receiver, thus obtaining $\mathbf{z}[l G+g]=\mathbf{F y}[l G+g]$, which by (5) yields

$$
\begin{array}{r}
\mathbf{z}[l G+g]=e^{j 2 \pi \varepsilon((l G+g) P+L) / N} \mathbf{\Phi} \mathbf{\Lambda}(\alpha \mathbf{S}[l] \mathbf{c}[g] \\
\left.+\mathbf{v}_{\mathrm{IMD}}[l G+g]\right)+\mathbf{z}_{\mathrm{WG}}[l G+g],
\end{array}
$$

where $\mathbf{\Phi}=\mathbf{F D F}^{H}$ is the $N \times N$ circulant matrix that models the ICI due to the CFO, $\mathbf{v}_{\text {IMD }}[l G+g]=\mathbf{F} \tilde{\mathbf{v}}_{\text {IMD }}[l G+g]$ is the IMD after the FFT operation, and $\mathbf{z}_{\mathrm{WG}}[l G+g]$ represents the AWGN.

Due to the spreading in the time domain, in order to decode the $N$ data symbols $\mathbf{s}_{k}[l]=\left[s_{1, k}[l] \cdots s_{N, k}[l]\right]^{T}$ relative to the $l$ th interval, the receiver of the $k$ th user has to collect $G$ consecutive vectors in (6). From (6), by defining the $N \times G$ matrix $\mathbf{Z}[l]=[\mathbf{z}[l G] \cdots \mathbf{z}[l G+G-1]]$, and $\mathbf{V}_{\mathrm{IMD}}[l]$ and $\mathbf{Z}_{\mathrm{WG}}[l]$ accordingly, we obtain

$$
\mathbf{Z}[l]=e^{j 2 \pi \varepsilon L / N} \mathbf{\Phi} \boldsymbol{\Lambda}\left(\alpha \mathbf{S}[l] \mathbf{C}+\mathbf{V}_{\mathrm{IMD}}[l]\right) \mathbf{E}[l]+\mathbf{Z}_{\mathrm{WG}}[l],
$$

where $\mathbf{C}=[\mathbf{c}[0] \cdots \mathbf{c}[G-1]]=\left[\begin{array}{llll}\mathbf{c}_{1} & \mathbf{c}_{2} & \cdots & \mathbf{c}_{K}\end{array}\right]^{T}$ is the rowwise $K \times G$ spreading code matrix, and $\mathbf{E}[l]$ is a $G \times G$ diagonal matrix defined as $[\mathbf{E}[l]]_{g+1, g+1}=e^{j 2 \pi \varepsilon(l G+g) P / N}$.

As in [3], we assume that the receiver is able to compensate both the phase-shift terms $\varphi=e^{j 2 \pi \varepsilon L / N} e^{j \pi \varepsilon(N-1) / N}$ and $\mathbf{E}[l]$. This hypothesis is clearly optimistic but has the merit to lead to BER performance lower bounds independently of any phase-estimation technique. Thus, by assuming perfect channel state information at the receiver side, the equalized data matrix can be constructed as $\mathbf{Z}_{\mathrm{EQ}}[l]=\varphi^{*} \alpha^{-1} \Lambda^{-1} \mathbf{Z}[l] \mathbf{E}[l]^{-1}$. Successively, after despreading, the vector $\mathbf{z}_{\mathrm{DS}, k}[l]=\mathbf{Z}_{\mathrm{EQ}}[l] \mathbf{c}_{k}^{*}$ contains the $N$ decision variables of the $k$ th user, as expressed by

$$
\mathbf{z}_{\mathrm{DS}, k}[l]=\mathbf{\Lambda}^{-1} \mathbf{M} \Lambda \mathbf{S}[l] \mathbf{C c}_{k}^{*}+\mathbf{z}_{\mathrm{DS}, k, \mathrm{IMD}}[l]+\mathbf{z}_{\mathrm{DS}, k, \mathrm{WG}}[l],
$$

where $\mathbf{M}=e^{-j \pi \varepsilon(N-1) / N} \boldsymbol{\Phi}$ is the phase-compensated ICI matrix, $\mathbf{z}_{\mathrm{DS}, k, \mathrm{IMD}}[l]=\alpha^{-1} \boldsymbol{\Lambda}^{-1} \mathbf{M} \mathbf{\Lambda} \mathbf{V}_{\mathrm{IMD}}[l] \mathbf{c}_{k}^{*}$ represents the IMD after equalization and despreading, and $\mathbf{z}_{\mathrm{DS}, k \text {, WG }}[l]=$ $\varphi^{*} \alpha^{-1} \boldsymbol{\Lambda}^{-1} \mathbf{Z}_{\mathrm{WG}}[l] \mathbf{E}[l]^{-1} \mathbf{c}_{k}^{*}$ stands for the AWGN. If we employ orthogonal spreading codes, the MAI is eliminated, because the product $\mathbf{S}[l] \mathbf{C} \mathbf{c}_{k}^{*}$ in (8) is equal to $\mathbf{s}_{k}[l]$. On the contrary, if the system is not capable to track and compensate for the time varying phase shifts summarized by $\mathbf{E}[l]$, we should take into account the introduction of MAI and of a mixed effect of MAI and ICI. Due to the lack of space, this will be the object of a future work.

\section{BER ANALYSIS}

By denoting with $\lambda_{n}=[\boldsymbol{\Lambda}]_{n, n}$ the channel gain of the $n$th subcarrier, the BER can be expressed by

$$
\begin{gathered}
(\mathrm{BER})_{n, k}=\int_{\lambda_{n}} P_{\mathrm{BE}, n, k}\left(\lambda_{n}\right) f_{\lambda_{n}}\left(\lambda_{n}\right) d \lambda_{n}, \\
P_{\mathrm{BE}, n, k}\left(\lambda_{n}\right)=\int_{\mathbf{S}, \bar{\lambda}_{n}} P_{\mathrm{BE}, n, k}(\mathbf{S}, \boldsymbol{\lambda}) f_{\bar{\lambda}_{n} \mid \lambda_{n}}\left(\bar{\lambda}_{n} \mid \lambda_{n}\right) f_{\mathbf{S}}(\mathbf{S}) d \mathbf{S} d \bar{\lambda}_{n},
\end{gathered}
$$

where $f_{\lambda_{n}}\left(\lambda_{n}\right)$ is the pdf of $\lambda_{n}, f_{\bar{\lambda}_{n} \mid \lambda_{n}}\left(\bar{\lambda}_{n} \mid \lambda_{n}\right)$ is pdf of

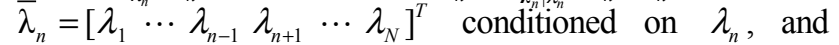
$P_{\mathrm{BE}, n, k}(\mathbf{S}, \boldsymbol{\lambda})$ is the BER conditioned on $\lambda$ and $\mathbf{S}[l]$. In order to find the BER, we firstly derive an approximated expression for the conditional probability in (10), and successively we average the approximated conditional BER over $f_{\lambda_{n}}\left(\lambda_{n}\right)$ as in (9). By multiplying (8) with $\lambda_{n}|\alpha|$, the scaled decision variable $z_{\mathrm{SC}, n, k}[l]$ can be expressed by

$$
\begin{aligned}
z_{\mathrm{SC}, n, k}[l]= & |\alpha| \mathbf{m}_{n}^{T} \boldsymbol{\Lambda} \mathbf{s}_{k}[l]+e^{-j \arg (\alpha)} \mathbf{m}_{n}^{T} \boldsymbol{\Lambda} \mathbf{V}_{\mathrm{IMD}}[l] \mathbf{c}_{k}^{*} \\
& +\varphi^{*} e^{-j \arg (\alpha)} \mathbf{z}_{n, \mathrm{WG}}[l]^{T} \mathbf{E}[l]^{-1} \mathbf{c}_{k}^{*},
\end{aligned}
$$

where $\mathbf{z}_{n, \mathrm{WG}}[l]^{T}$ is the $n$th row of $\mathbf{Z}_{\mathrm{WG}}[l]$, and $\mathbf{m}_{n}=$ $\left[\begin{array}{lll}m_{n, 1} & \cdots & m_{n, N}\end{array}\right]^{T}$ is the $n$th row of $\mathbf{M}$.

By dropping the index $l$, (11) becomes

$$
\begin{aligned}
& z_{\mathrm{SC}, n, k}=|\alpha| m \lambda_{n} s_{n, k}+|\alpha| \sum_{n^{\prime}=1, n^{\prime} \neq n}^{N} m_{n, n^{\prime}} \lambda_{n^{\prime}} s_{n^{\prime}, k} \\
& +\sum_{g=0}^{G-1} e^{-j \arg (\alpha)} c_{k, g}^{*} \sum_{n^{\prime}=1}^{N} m_{n, n^{\prime}} \lambda_{n^{\prime}}, v_{\mathrm{IMD}, n^{\prime}, g}+z_{\mathrm{AWGN}, n, k},
\end{aligned}
$$

where $m=m_{n, n}, v_{\mathrm{IMD}, n, g}$ is the $(n, g)$ th element of $\mathbf{V}_{\mathrm{IMD}}$, and $z_{\mathrm{AWGN}, n, k}=\varphi^{*} e^{-j \arg (\alpha)} \mathbf{z}_{n, \mathrm{WG}}^{*} \mathbf{E}^{-1} \mathbf{c}_{k}^{*}$. By constructing the conditional random variable $t_{\mathrm{SC}, n, k}=z_{\mathrm{SC}, n, k} \mid \lambda_{n}$, we can express (12) as a function of the random variables $\left\{\hat{\lambda}_{n^{\prime}, n}\right\}$, where $\hat{\lambda}_{n^{\prime}, n}=\lambda_{n^{\prime}} \mid \lambda_{n}$ indicates the $n^{\prime}$ th subcarrier channel gain conditioned on the $n$th subcarrier channel gain. The $(N-1) \times 1$ vector $\hat{\lambda}_{n}=\left[\hat{\lambda}_{1, n} \cdots \hat{\lambda}_{n-1, n} \hat{\lambda}_{n+1, n} \cdots \hat{\lambda}_{N, n}\right]^{T}$ is still a Gaussian random vector, with mean value $\boldsymbol{\eta}_{\hat{\lambda}}=E\left\{\hat{\boldsymbol{\lambda}}_{n}\right\}$ and covariance $\mathbf{R}_{\hat{\lambda}_{n}}=E\left\{\hat{\boldsymbol{\lambda}}_{n} \hat{\boldsymbol{\lambda}}_{n}^{H}\right\}$ expressed by [8]

$$
\boldsymbol{\eta}_{\hat{\lambda}_{n}}=\lambda_{n} r_{\lambda_{n} \lambda_{n}}^{-1} \mathbf{r}_{\bar{\lambda}_{n} \lambda_{n}}, \quad \mathbf{R}_{\hat{\lambda}_{n}}=\mathbf{R}_{\bar{\lambda}_{n}}-r_{\lambda_{n} \lambda_{n}}^{-1} \mathbf{r}_{\bar{\lambda}_{n} \lambda_{n}} \mathbf{r}_{\bar{\lambda}_{n} \lambda_{n}}^{H},
$$

where $r_{\lambda_{n}, \lambda_{n}}=E\left\{\lambda_{n}, \lambda_{n}^{*}\right\}$ is the statistical correlation between the channels on the $n^{\prime}$ th and the $n$th subcarrier, $\mathbf{r}_{\bar{\lambda}_{n} \lambda_{n}}=E\left\{\overline{\boldsymbol{\lambda}}_{n} \lambda_{n}^{*}\right\}$ is the crosscorrelation vector between the channel of interest and the other channels, and $\mathbf{R}_{\bar{\lambda}_{n}}=E\left\{\overline{\boldsymbol{\lambda}}_{n} \overline{\boldsymbol{\lambda}}_{n}^{H}\right\}$ is the crosscorrelation matrix of the other channels. Consequently, defining the $(N-1) \times 1$ zero-mean Gaussian random vector $\boldsymbol{\pi}_{n}=\hat{\boldsymbol{\lambda}}_{n}-\boldsymbol{\eta}_{\hat{\lambda}_{n}}=$ $\left[\begin{array}{llllll}\pi_{1, n} & \cdots & \pi_{n-1, n} & \pi_{n+1, n} & \cdots & \pi_{N, n}\end{array}\right]^{T}, t_{\mathrm{SC}, n, k}$ becomes

$$
t_{\mathrm{SC}, n, k}=\lambda_{n}\left(|\alpha| m s_{n, k}+z_{1, n, k}\right)+z_{2, n, k},
$$

where 


$$
\begin{gathered}
z_{1, n, k}=z_{\mathrm{ICI}, n, k}+z_{\mathrm{IMD}, n, k}, \\
z_{2, n, k}=\tilde{z}_{\mathrm{ICI}, n, k}+\tilde{z}_{\mathrm{IMD}, n, k}+z_{\mathrm{AWGN}, n, k}, \\
z_{\mathrm{ICI}, n, k}=|\alpha| r_{\lambda_{n} \lambda_{n}}^{-1} \sum_{n^{\prime}=1, n^{\prime} \neq n}^{N} m_{n, n} r_{\lambda_{n^{\prime}} \lambda_{n}} s_{n^{\prime}, k}, \\
z_{\mathrm{IMD}, n, k}=e^{-j \arg (\alpha)} r_{\lambda_{n} \lambda_{n}}^{-1} \sum_{g=0}^{G-1} c_{k, g}^{*} \sum_{n^{\prime}=1}^{N} m_{n, n} r_{\lambda_{n^{\prime}} \lambda_{n}} v_{\mathrm{IMD}, n^{\prime}, g}, \\
\tilde{z}_{\mathrm{ICI}, n, k}=|\alpha| \sum_{n^{\prime}=1, n^{\prime} \neq n}^{N} m_{n, n} \pi_{n^{\prime}, n} S_{n^{\prime}, k}, \\
\tilde{z}_{\mathrm{IMD}, n, k}=e^{-j \arg (\alpha)} \sum_{g=0}^{G-1} c_{k, g}^{*} \sum_{n^{\prime}=1, n^{\prime} \neq n}^{N} m_{n, n} \pi_{n^{\prime}, n} v_{\mathrm{IMD}, n^{\prime}, g} .
\end{gathered}
$$

The noisy term $\lambda_{n} z_{1, n, k}$ in (14) is proportional to $\lambda_{n}$, and hence it represents the interference that fades coherently with the useful signal, while $z_{2, n, k}$ represents the interference that fades independently of $\lambda_{n}$, because the powers of the terms in (19) and (20) do not depend on the specific channel coefficient $\lambda_{n}$. Consequently, the conditional signal-to-interference plus noise ratio can be expressed as

$$
\gamma_{n, k}\left(\lambda_{n}\right)=\frac{\left|\lambda_{n}\right|^{2}|\alpha m|^{2} \sigma_{\mathrm{S}}^{2}}{\left|\lambda_{n}\right|^{2} \sigma_{1, n, k}^{2}+\sigma_{2, n, k}^{2}},
$$

where

$$
\begin{aligned}
& \sigma_{1, n, k}^{2}=\sigma_{\mathrm{ICI}, n, k}^{2}+\sigma_{\mathrm{IMD}, n, k}^{2}, \\
& \sigma_{2, n, k}^{2}=\tilde{\sigma}_{\mathrm{ICI}, n, k}^{2}+\tilde{\sigma}_{\mathrm{IMD}, n, k}^{2}+\sigma_{\mathrm{AWGN}}^{2}, \\
& \sigma_{\mathrm{ICl}, n, k}^{2}=\left|\alpha r_{\lambda_{n} \lambda_{n}}^{-1}\right|^{2} \sum_{n^{\prime}=1, n^{\prime} \neq n}^{N}\left|m_{n, n^{\prime}}, r_{\lambda_{n} \lambda_{n}}\right|^{2} \sigma_{\mathrm{S}}^{2}, \\
& \sigma_{\mathrm{IMD}, n, k}^{2}=r_{\lambda_{n} \lambda_{n}}^{-2} \sum_{g=0}^{G-1} \sum_{g^{\prime}=0}^{G-1} c_{k, g}^{*} c_{k, g^{\prime}} \overline{\mathbf{m}}_{n}^{T} \mathbf{R}_{\mathbf{v}}\left(g, g^{\prime}\right) \overline{\mathbf{m}}_{n}^{*}, \\
& \tilde{\sigma}_{\mathrm{ICI}, n, k}^{2}=|\alpha|^{2} \sum_{n^{\prime}=1, n^{\prime} \neq n}^{N}\left|m_{n, n^{\prime}}\right|^{2}\left(r_{\lambda_{n^{\prime}} \lambda_{n}}-r_{\lambda_{n} \lambda_{n}}^{-1}\left|r_{\lambda_{n}, \lambda_{n}}\right|^{2}\right) \sigma_{\mathrm{S}}^{2}, \\
& \tilde{\sigma}_{\mathrm{IMD}, n, k}^{2}=\sum_{g=0}^{G-1} \sum_{g^{\prime}=0}^{G-1} c_{k, g}^{*} c_{k, g^{\prime}} . \\
& \sum_{n^{\prime}=1, n^{\prime} \neq n}^{N} \sum_{n^{\prime \prime}=1, n^{\prime \prime} \neq n}^{N} m_{n, n^{\prime}}, m_{n, n^{n}}^{*}\left[\mathbf{J}_{n}^{T} \mathbf{R}_{\aleph_{\aleph_{n}}} \mathbf{J}_{n}\right]_{n^{\prime}, n^{n}}\left[\mathbf{R}_{\mathbf{v}}\left(g, g^{\prime}\right)\right]_{n^{\prime}, n^{\prime \prime}},
\end{aligned}
$$

where $\left[\overline{\mathbf{m}}_{n}\right]_{n^{\prime}}=m_{n, n}, r_{\lambda_{n}, \lambda_{n}}$, the $(N-1) \times N$ matrix $\mathbf{J}_{n}$ is obtained from $\mathbf{I}_{N}$ by removing the $n$th row, and $\mathbf{R}_{\mathbf{v}}\left(g, g^{\prime}\right)$ is expressed by

$$
\mathbf{R}_{\mathbf{v}}\left(g, g^{\prime}\right)=E\left\{\mathbf{v}_{\mathrm{IMD}, g} \mathbf{v}_{\mathrm{IMD}, g^{\prime}}^{H}\right\}=\mathbf{F R}_{\mathrm{CP}} \mathbf{R}_{\overline{\mathrm{v}}}\left(g, g^{\prime}\right) \mathbf{R}_{\mathrm{CP}}^{T} \mathbf{F}^{H},
$$

which can be calculated by exploiting the knowledge of the IMD covariance matrix.

At this point, we approximate as Gaussian the interference terms in (15) and (16). Such an approximation is reasonable under the hypothesis of a high number of subcarriers. Note that for constant-modulus constellations the term in (19) is exactly Gaussian. Assuming for simplicity 4-QAM with Gray coding, the conditional BER is expressed by

$$
P_{\mathrm{BE}, n, k}\left(\lambda_{n}\right)=Q\left(\sqrt{\gamma_{n, k}\left(\lambda_{n}\right)}\right),
$$

and the resultant BER is obtained by inserting (29), (21), and the Rayleigh pdf of $\left|\lambda_{n}\right|$ in (9), with $\sigma_{\lambda}^{2}=E\left\{\left|\lambda_{n}\right|^{2}\right\}$. The final BER can therefore be obtained as [9]

$$
\begin{aligned}
& (\mathrm{BER})_{n, k}=\int_{0}^{+\infty} Q\left(\sqrt{\gamma\left(\lambda_{n}\right)}\right) \frac{2\left|\lambda_{n}\right|}{\sigma_{\lambda}^{2}} e^{-\frac{\left|\lambda_{n}\right|^{2}}{\sigma_{\lambda}^{2}}} d\left|\lambda_{n}\right| \\
& =\frac{1}{2}-\frac{\sqrt{2 \mu_{n, k}^{2}}}{4} e^{-\frac{\mu_{n, k}^{2}}{2 v_{n, k}^{2}}} \sum_{i=0}^{+\infty} \frac{1}{i !}\left(\frac{\mu_{n, k}^{2}}{2 v_{n, k}^{2}}\right)_{2}^{i} F_{0}\left(i+\frac{3}{2}, \frac{1}{2} ; ;-v_{n, k}^{2}\right),
\end{aligned}
$$

where ${ }_{p} F_{q}$ denotes the generalized hypergeometric function, $\mu_{n, k}^{2}=|\alpha m|^{2} \sigma_{\mathrm{S}}^{2} \sigma_{\lambda}^{2} \sigma_{2, n, k}^{-2}$ and $v_{n, k}^{2}=\sigma_{1, n, k}^{2} \sigma_{\lambda}^{2} \sigma_{2, n, k}^{-2}$.

\section{SIMULATION RESULTS}

We consider a 4-QAM MC-DS-CDMA system with $N=256, \Delta_{f}=1 / T=156.25 \mathrm{kHz}$, cyclic prefix $L=64$, a channel characterized by an exponentially decaying power delay profile and rms delay spread of $250 \mathrm{~ns}$, and a perfectly predistorted amplifier [6]. We also assume that the base station employs Walsh-Hadamard (WH) spreading codes of length $G=16$. The received $E_{b} / N_{0}$ is defined before the despreading, while the BER is averaged over all the subcarriers and users.

Fig. 1 shows the BER versus $E_{b} / N_{0}$ for different values of the normalized CFO $\varepsilon$, assuming linear amplification at the transmitter (i.e., without IMD). The good agreement between theoretical analysis and simulated BER for all practical values of $\varepsilon$ is evident. This fact clearly testifies that the Gaussian approximation of the ICI leads to accurate results in frequency-selective scenarios.

Fig. 2 shows the impact of the HPA on the BER performance in the absence of CFO. We defined $\mathrm{OBO}=P_{\mathrm{U}, \mathrm{MAX}} / \sigma_{\mathrm{U}}^{2}$, where $P_{\mathrm{U}, \mathrm{MAX}}$ and $\sigma_{\mathrm{U}}^{2}$ are the maximum power and the mean power, respectively, of the HPA output signal. We assume that the amplifier is perfectly predistorted, i.e., it behaves as a clipper of the HPA input envelope. Fig. 2 suggests that the Gaussian approximation of the IMD is slightly worse than for the ICI in Fig. 1, especially at the saturating BER. Indeed, for a clipping amplifier, the Gaussian approximation of the IMD is very accurate only for moderate SNR and for high number of subcarriers. However, in the assumed scenario the BER mismatch seems to be quite small.

In Fig. 3, we still assume $\varepsilon=0$, and we focus on the impact of the number $K$ of active users on the BER performance. We also assume that each user employs a fixed spreading code, i.e., that the $k$ th user employs the $k$ th row of the Hadamard matrix. Fig. 3 shows that at low SNR the BER increases with the number of active users, as expected. However, the BER floor at high SNR does not increase with the number of users. Such a behavior is due to the fact that the IMD powers in (25) and (27) depend on $\mathbf{R}_{\mathbf{v}}\left(g, g^{\prime}\right)$, which is highly sensitive to the choice of the spreading codes among those provided by the Hadamard matrix.

Fig. 4 shows the joint effect of CFO and HPA on the BER performance when $\varepsilon=0.02$. The results show that, in the presence of a CFO, increasing the output HPA power back-off 
at the transmitter beyond a certain value (that obviously depends on $\varepsilon$ ), does not help too much in reducing the BER because the IMD reduction is masked by the presence of the CFO induced ICI.

\section{CONCLUSION}

In this paper, we have evaluated the BER of 4-QAM MC-DSCDMA downlink systems subject to both CFO and IMD in frequency-selective Rayleigh fading channels. The BER analysis is quite accurate in several conditions. The analysis can easily be extended to $M$-QAM and $M$-PSK constellations. Future works will analyze the effects of an imperfect phase shift compensation at the receiver side and may also focus on channel estimation errors and channel coding effects.

\section{REFERENCES}

[1] S. Hara and R. Prasad, "Overview of multicarrier CDMA," IEEE Commun. Mag., vol. 35, pp. 126-133, Dec. 1997.

[2] T. Pollet, M. van Bladel, and M. Moeneclaey, "BER sensitivity of OFDM systems to carrier frequency offset and Wiener phase noise," IEEE Trans. Commun., vol. 43, pp. 191-193, Feb./Mar./Apr. 1995.

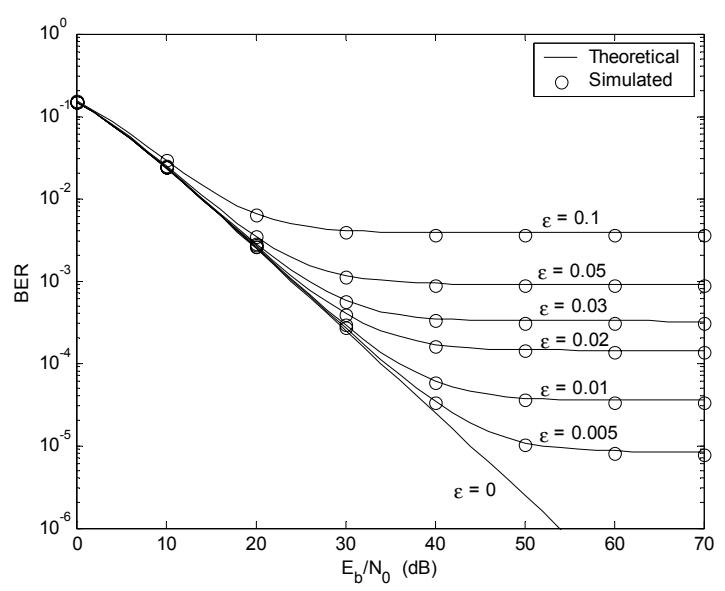

Fig. 1. Impact of the CFO $\varepsilon$ ( $K=16$, no IMD).

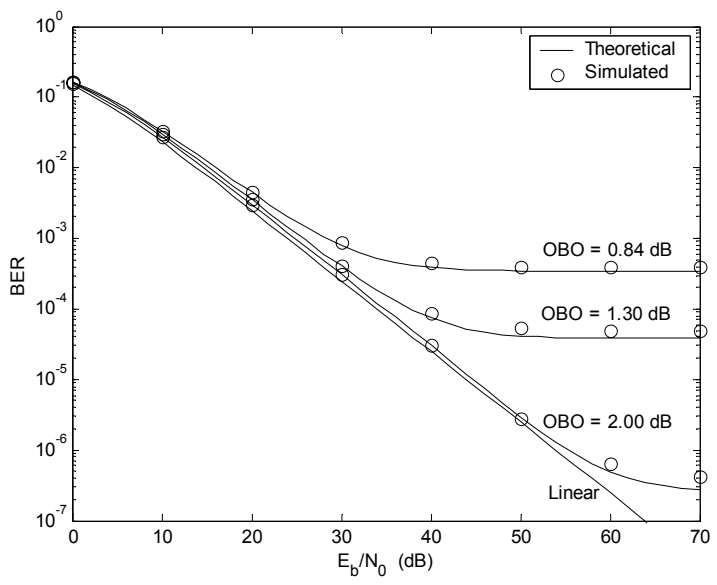

Fig. 2. Impact of the HPA ( $K=16$, no CFO).
[3] H. Steendam and M.E. Moeneclaey, "The sensitivity of downlink MC-DS-CDMA to carrier frequency offsets," IEEE Commun. Lett., vol. 5, pp. 215-217, May 2001.

[4] J.H. Jong and W.E. Stark, "Performance analysis of coded multicarrier spread-spectrum systems in the presence of multipath fading and nonlinearities," IEEE Trans. Commun., vol. 49, pp. 168-179, Jan. 2001.

[5] Z. Wang and G.B. Giannakis, "Wireless multicarrier communications: where Fourier meets Shannon," IEEE Signal Processing Mag., vol. 17, pp. 29-48, May 2000.

[6] P. Banelli and S. Cacopardi, "Theoretical analysis and performance of OFDM signals in nonlinear AWGN channels," IEEE Trans. Commun., vol. 48, pp. 430-441, Mar. 2000.

[7] X. Ma, C. Tepedelenlioğlu, G.B. Giannakis, and S. Barbarossa, "Non-data-aided carrier offset estimators for OFDM with null subcarriers: identifiability, algorithms, and performance," IEEE JSAC, vol. 19, pp. 2504-2515, Dec. 2001.

[8] S.M. Kay, Fundamentals of Statistical Signal Processing: Estimation Theory, vol. 1, Prentice Hall, 1993.

[9] L. Rugini and P. Banelli, "Symbol error probability of linearly modulated signals affected by Gaussian interference in Rayleigh channels," Tech. Rep. RT-005-03, Dept. Elect. Inform. Eng., Perugia, Italy, Dec. 2003. Available: http://www.diei.unipg.it/rt/DIEI_RT.htm .

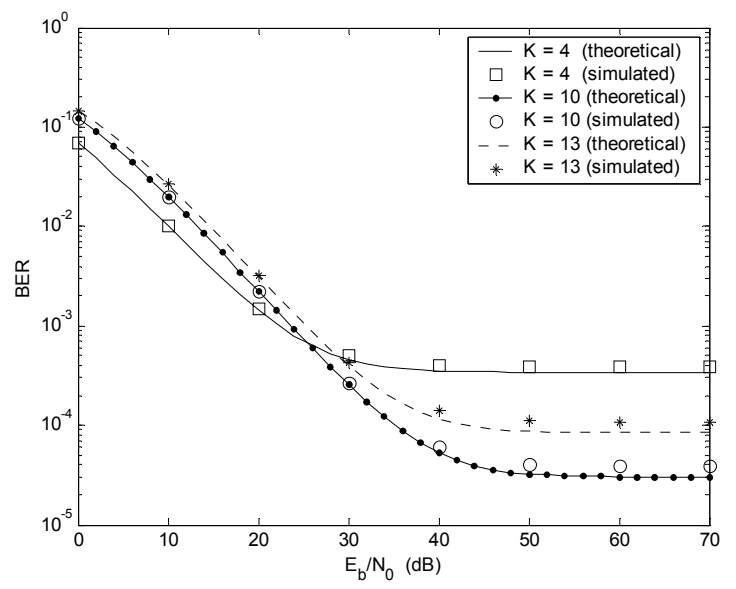

Fig. 3. Impact of \# of users $K(\mathrm{OBO}=0.84 \mathrm{~dB}$, no $\mathrm{CFO})$.

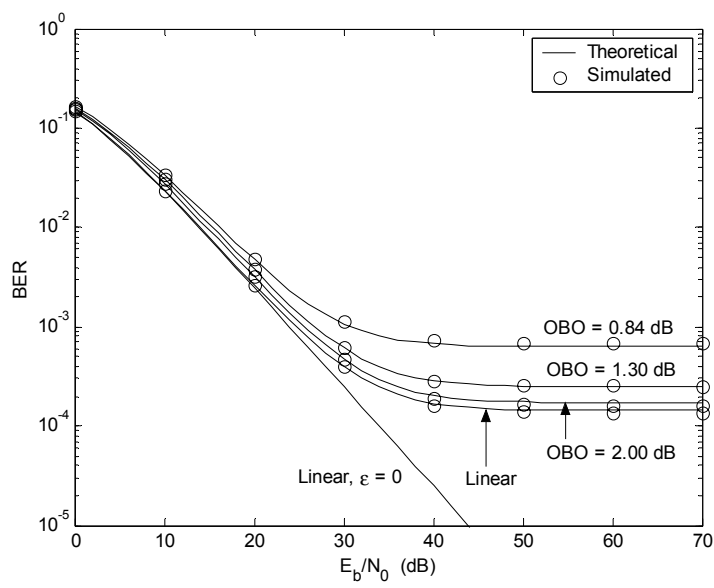

Fig. 4. Impact of both $\mathrm{CFO}$ and $\mathrm{HPA}(K=16, \varepsilon=0.02)$. 